\title{
Retraction Notice
}

Title of retracted article: Author(s):

* Corresponding author. Journal:

Year:

Volume:

Number:

Pages (from - to):

DOI (to PDF):

Paper ID at SCIRP:

Article page:

Retraction date:
A Numerical Study on the Charging Voltage of the Lunar Surface Zhihao Wang, Dongbo Tian, Yuming Liu, Yenan Liu, Yigang Ding, Zicai Shen

Email:singyareqq.com

Journal of Applied Mathematics and Physics 2019

7

12

$3177-3185$

https://doi.org/10.4236/jamp.2019.712222

97313

http: //www.scirp.org/Journal/PaperInformation.aspx?PaperID=97313 2019-12-24

Retraction initiative (multiple responses allowed; mark with $\mathbf{X}$ ):

X All authors

$\square$ Some of the authors:

Editor with hints from

Journal owner (publisher)

O Institution:

O Reader:

Other:

Date initiative is launched:

$$
\text { 2019-12-24 }
$$

Retraction type (multiple responses allowed):

$\square$ Unreliable findings
Lab error
Inconsistent data
Analytical error
O Biased interpretation

O Other:

Irreproducible results

$\square$ Failure to disclose a major competing interest likely to influence interpretations or recommendations

Unethical research

$\square$ Fraud

Data fabrication

Plagiarism

Fake publication

$\square$ Self plagiarism

Other:

Copyright infringement

$\square$ Other legal concern:

Editorial reasons

$\mathrm{O}$ Handling error

Unreliable review(s)

Decision error

Other:

$\mathbf{X}$ Other: Request from the Authors.

Results of publication (only one response allowed):

$\square$ are still valid.

$\mathbf{X}$ were found to be overall invalid.

Author's conduct (only one response allowed):

$\square$ honest error

$\square$ academic misconduct

$\mathbf{X}$ none (not applicable in this case - e.g. in case of editorial reasons)

* Also called duplicate or repetitive publication. Definition: "Publishing or attempting to publish substantially the same work more than once." 


\section{History}

Expression of Concern:

$\square$ yes, date: yyyy-mm-dd

$X$ no

\section{Correction:}

$\square$ yes, date: yyyy-mm-dd

$X$ no

\section{Comment:}

This article has been retracted to straighten the academic record. In making this decision the Editorial Board follows COPE's Retraction Guidelines. Aim is to promote the circulation of scientific research by offering an ideal research publication platform with due consideration of internationally accepted standards on publication ethics. The Editorial Board would like to extend its sincere apologies for any inconvenience this retraction may have caused. 


\title{
A Numerical Study on the Charging Voltage of the Lunar Surface
}

\author{
Beijing Institute of Spacecraft Environment Engineering, Beijing, China \\ Email: singyar@qq.com
}

Zhihao Wang, Dongbo Tian, Yuming Liu, Yenan Liu, Yigang Ding, Zicai Shen

How to cite this paper: Wang, Z.H., Tian, D.B., Liu, Y.M., Liu, Y.N., Ding, Y.G. and Shen, Z.C. (2019) A Numerical Study on the Charging Voltage of the Lunar Surface. Journal of Applied Mathematics and Physics, 7, 3177-3185.

https://doi.org/10.4236/jamp.2019.712222

Received: November 25, 2019

Accepted: December 17, 2019

Published: December 24, 2019

\section{Abstract}

Under the influence of solar light and space plasma, the charging phenomenon will occur on the lunar surface. According to the principle of current balance, a one-dimensional charging model of the lunar surface is established, and the variation of the charging voltage of the lunar surface with the solar zenith angle and plasma parameters are calculated. The results show that with the increase of solar zenith angle, the charging potential of the lunar surface gradually transits from a positive potential to a negative potential at the terminators, then reaches the maximum value of the negative potential in the lunar night area; among them, the charging voltage in the lunar day area is affected by a number of plasma parameters, and the charging voltage in the lunar night area is greatly affected by the electron temperature. According to the relative position of the moon and the earth, the moon will experience different plasma environments, and its charging voltage will change periodically in a lunar day. In the lunar night area, because of the gradual change and transformation of the plasma environments, the charging potential will change accordingly. When passing through different plasma environments, the charging potential may also change abruptly in a short time.

\section{Keywords}

Lunar Surface Charging, Plasma, Current Balance Equation

\section{Introduction}

Before humans landed on the moon, researchers predicted that the day side of the moon would be positively charged due to photoelectron emission [1] [2]. This prediction was subsequently confirmed by the SIDE experimental device deployed in Apollo project. The measurement results show that in the solar wind, the moon surface is positively charged $(\approx+10 \mathrm{~V})$ in the daytime, negatively charged $(\approx-100 \mathrm{~V})$ near the terminators and at night [3]. The lunar surface po- 
tential measured by electron reflectometer (ER) on the Lunar Prospector (LP) is basically the same as that observed by SIDE [4] [5]. However, under special environmental conditions, such as during the solar high energy particle (SEP) event, the potential of the lunar surface may be as high as $\sim 4 \mathrm{kV}$ [6] [7].

The main current sources leading to lunar surface charging include the electron light emission caused by the solar ultraviolet (UV) radiation, the plasma electron and ion current, and the secondary electron current on the lunar surface. When the sum of the current in and out of the lunar surface is zero, the lunar surface reaches the equilibrium potential, which is actually the basic principle of calculating the lunar surface potential. It is worth noting that the calculation results of "current balance" method are roughly consistent with the actual observation results of the lunar surface [8] [9].

The charging of the lunar surface has a significant and extensive impact on the lunar surface environment and lunar exploration. First of all, the charging of the lunar surface is considered to be one of the main causes of dust lofting and transportation on the lunar surface 110]. The lunar ejecta and meteorites experiment (LEAM) device deployed on the lunar surface in Apollo mission confirmed the charging phenomenon of the lunar surface. Through response modeling analysis [11] and experimental research [12], the device shows that the recorded impact can very well come from the low-speed charged dust particles, and the event occurrence time is concentrated in the terminators [13]. Secondly, the charging of the lunar surface will have a significant impact on the lunar exploration activities. The charging of the lunar surface will lead to a strong adhesion of the lunar dust. Apollo astronauts have experienced problems such as seal wear and pollution, which are all aggravated by the electrostatic process [14] \{15]. In addition, there is a large difference in the charging properties between the lunar detector and lunar soil, which may lead to different charging, electrostatic discharge (ESD) may occur and damage the electronic equipments [16] [17].

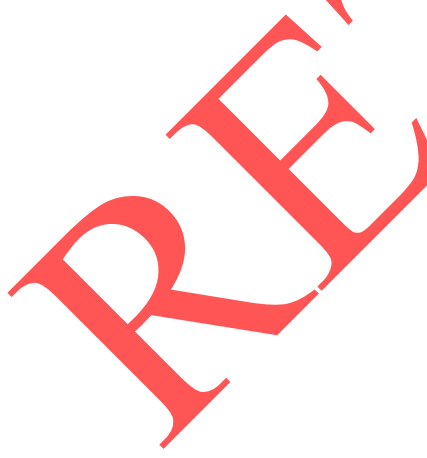

In order to analyze the variation of the charging voltage with the key envirommental parameters, a one-dimensional charging model is established based on the current balance equation. In addition to the typical solar wind plasma and solar radiation environment, this paper also considers the environmental factors such as the lunar wake, the earth's magnetosheath and the magnetotail experienced in the whole lunar daily cycle, and gives the simplified charging voltage with the change of the lunar local time. In Section 2, we first establish a one-dimensional charging model of the lunar surface and give the calculation equation used in the model. The corresponding numerical calculation and analysis results are given in Section 3, including the charging characteristics varying with the solar zenith angle, plasma parameters and time. Finally, a brief summary and conclusion are given in Section 4.

\section{Charging Model}

Because the scale of the moon is much larger than that of the sheath formed by 
the charging of the lunar surface, the charging problem of the lunar surface can be simplified to the solution of one-dimensional current balance equation. As shown in Equation (1).

$$
J_{p}+J_{e}+J_{i}+J_{s}=0
$$

where $J_{p}$ is the photocurrent density, $J_{e}$ is the plasma electron current density, $J_{i}$ is the plasma ion current density, $J_{s}$ is the secondary electron current density.

Considering the extremely low conductivity of the lunar dust and low plasma energy, we can ignor the secondary electrons caused by the incident plasma electrons and ions (the secondary electron emission coefficient of lunar soil reaches the peak value at about $700 \mathrm{eV}$ [18]), the Equation (1) in the equilibrium state can be simplified as follows:

$$
J_{p}+J_{e}+J_{i}=0
$$

In the moon night, it can be further simplified as:

$$
J_{e}+J_{i}=0
$$

According to the current density equation given in the appendix of reference [19]:

When $\phi_{0}>0$ :

$$
J_{p}=-e n_{p 0}\left(\frac{k_{B} T_{p}}{2 \pi m_{e}}\right)^{1 / 2} \exp \left(-\frac{\left|e \phi_{0}\right|}{k_{B} T_{p}}\right)
$$

where $\phi_{0}$ is the funar surface potential in equilibrium, $k_{B}$ is the Boltzmann constant, $T_{p}$ is the photoelectron temperature, $e$ is the electron charge, $n_{p 0}$ is the photoelectron density at the surface, $T_{e}$ is the plasma electron temperature, $m_{e}$ is the electron mass.

$$
\text { When } \phi_{0}<0 \text { : }
$$

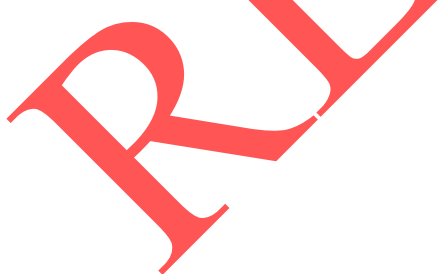

$$
\begin{gathered}
J_{p}=-e n_{p 0}\left(\frac{k_{B} T_{p}}{2 \pi m_{e}}\right)^{1 / 2} \\
n_{p 0}=-\left(\frac{2 \pi m_{e}}{k_{B} T_{p}}\right)^{1 / 2} \frac{J_{P 0} \cos \theta}{\mathrm{e}}
\end{gathered}
$$

where $\theta$ is the solar zenith angle, $J_{p 0}$ is the current density of photoelectron perpendicular to the surface.

When $\phi_{0}>0$ :

$$
\begin{gathered}
J_{e}=e n_{0}\left\{\left(\frac{k_{B} T_{e}}{2 \pi m_{e}}\right)^{1 / 2} \exp \left[-\frac{m_{e}(V \cos \theta)^{2}}{2 k_{B} T_{e}}\right]+\frac{V \cos \theta}{2}\left(\operatorname{erf}\left[\left(\frac{m_{e}}{2 k_{B} T_{e}}\right)^{1 / 2} V \cos \theta\right]-1\right)\right\} \\
J_{i}=-e n_{0}\left\{\left(\frac{k_{B} T_{i}}{2 \pi m_{i}}\right)^{1 / 2} \exp \left(-\frac{m_{i}}{2 k_{B} T_{i}}\left[V \cos \theta+\left(\frac{2 e \phi_{0}}{m_{i}}\right)^{1 / 2}\right]^{2}\right)\right. \\
\left.+\frac{V \cos \theta}{2}\left(\operatorname{erf}\left(\left[\frac{m_{i}}{2 k_{B} T_{i}}\right]^{1 / 2}\left[V \cos \theta+\left(\frac{2 e \phi_{0}}{m_{i}}\right)^{1 / 2}\right]\right)-1\right)\right\}
\end{gathered}
$$


where $V$ is the plasma overall velocity, $n_{0}$ is the plasma density outside the sheath, $m_{i}$ is the proton mass.

When $\phi_{0}<0$ :

$$
\begin{gathered}
J_{i}=-e n_{0}\left\{\left(\frac{k_{B} T_{i}}{2 \pi m_{i}}\right)^{1 / 2} \exp \left[-\frac{m_{i}(V \cos \theta)^{2}}{2 k_{B} T_{i}}\right]\right. \\
\left.+\frac{V \cos \theta}{2}\left(\operatorname{erf}\left[\left(\frac{m_{i}}{2 k_{B} T_{i}}\right)^{1 / 2} V \cos \theta\right]-1\right)\right\} \\
J_{e}=e n_{0}\left\{\left(\frac{k_{B} T_{e}}{2 \pi m_{e}}\right)^{1 / 2} \exp \left(-\frac{m_{e}}{2 k_{B} T_{e}}\left[V \cos \theta+\left(-\frac{2 e \phi_{0}}{m_{e}}\right)^{1 / 2}\right]^{2}\right)\right. \\
\left.+\frac{V \cos \theta}{2}\left(\operatorname{erf}\left(\left[\frac{m_{e}}{2 k_{B} T_{e}}\right]^{1 / 2}\left[V \cos \theta\left(-\frac{2 e \phi_{0}}{m_{e}}\right)^{1 / 2}\right]\right)-1\right)\right\}
\end{gathered}
$$

According to Equation (1) to Equation (10), the variation of $\phi_{0}$ with different parameters can be calculated.

\section{Model Results and Analysis}

\subsection{Influence of Parameters}

There are many environmental factors that affect the charging voltage of the lunar surface, among which $\theta$ represents the illumination characteristics, which are related to the position coordinates of the lunar surface and the local time of the moon; $n_{0}, T_{e}, T_{p}, T_{p}$ and $V$ represent the plasma parameters of the lunar surface, which are related to the solar activity and the relative position of the earth and the moon. In addition, there is also $J_{p 0}$ which reflects the photoluminescence characteristics of lunar soil, and is related to the physical and chemical properties of lunar soil.

Change the value of $n_{0}$, and calculate the changing rule of $\phi_{0}$ with $\theta$ as shown in Figure 1, where $\theta$ also corresponds to the lunar local time. Without considering the location coordinate, $\theta=0^{\circ}$ corresponds to the noon (local time $T_{L}=$ 12 ), when $\theta=90=$ corresponds to the evening $\left(T_{L}=18\right)$. With the increase of $\theta$, the charging potential of the lunar surface gradually transits from the positive potential to the zero potential at the terminators. With the increase of $\theta$, the influence of photoelectron gradually weakens. The plasma electron current gradually dominates the current balance process and the lunar surface has negative potential. When $\theta>90^{\circ}$, the lunar surface is charged to the maximum negative value.

It can be seen from the analysis of the curves corresponding to different $n_{0}$ values that, with the increase of $n_{0}$ value, the charging voltage in the day area decreases gradually. When $n_{0}=100 / \mathrm{cm}^{3}$, the charging voltage in the day area is negative, at the same time, with the increase of $n_{0}$ value, the smaller the value of $\theta$ corresponding to the zero potential of the lunar surface is, indicating the earlier the zero potential occurs. 


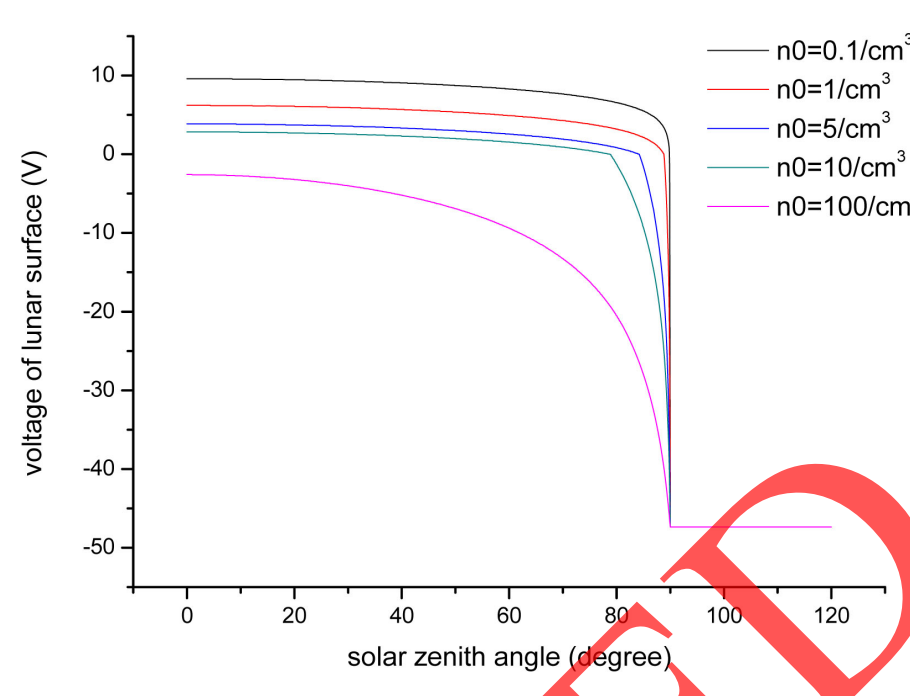

Figure 1. Variation of $\phi_{0}$ with $\theta$ ( $n_{0}$ changing).

Change the $T_{e}$ value and calculate the changing rule of $\phi_{0}$ with $\theta$ as shown in Figure 2. With the increase of $T_{e}$ value, the charging voltage in the day area decreases gradually, and the charging voltage in the night side is more negative (the absolute value increases).

Change the $T_{p}$ value, and calculate the change rule of $\phi_{0}$ with $\theta$ as shown in Figure 3. With the increase of $T_{p}$ value, the charging voltage in the day region decreases gradually. Obviously, $T_{p}$ value has no effect on the charging in the region of moon night.

Change the $V$ value, and calculate the changing rule of $\phi_{0}$ with $\theta$ as shown in Figure 4. With the increase of $V$ value, the charging voltage of the day area decreases gradually.

\subsection{Charging Voltage Changing with Time}

In fact, the charging environment of the lunar surface changes periodically with the change of the local time of the moon. Since lunar local time 12 o'clock, the lunar surface will successively experience the influence of solar wind plasma, lunar wake plasma, magnetic sheath plasma and magnetic tail plasma. There are certain differences in plasma parameters, resulting in periodic fluctuation of charging voltage, as shown in Figure 5.

As shown in Figure 5, when $T_{L}<18$, the surface charging is mainly affected by solar wind plasma and solar light. When $T_{L}>18$, the surface charging gradually transits to be affected by the plasma in the wake of the moon, and the charging voltage is further reduced. When $T_{L}>20.82$ (based on the estimation of the proportion of each plasma environment time experienced in a lunar day), the surface charging gradually transits to be affected by the plasma in the magnetic sheath The charging voltage rises to about $-325 \mathrm{~V}$; when $T_{L}>22.42$, the charging voltage of the surface gradually changes to about $-340 \mathrm{~V}$ due to the influence of the plasma in the magnetic tail. The plasma parameters selected in the calculation are shown in Table 1. 


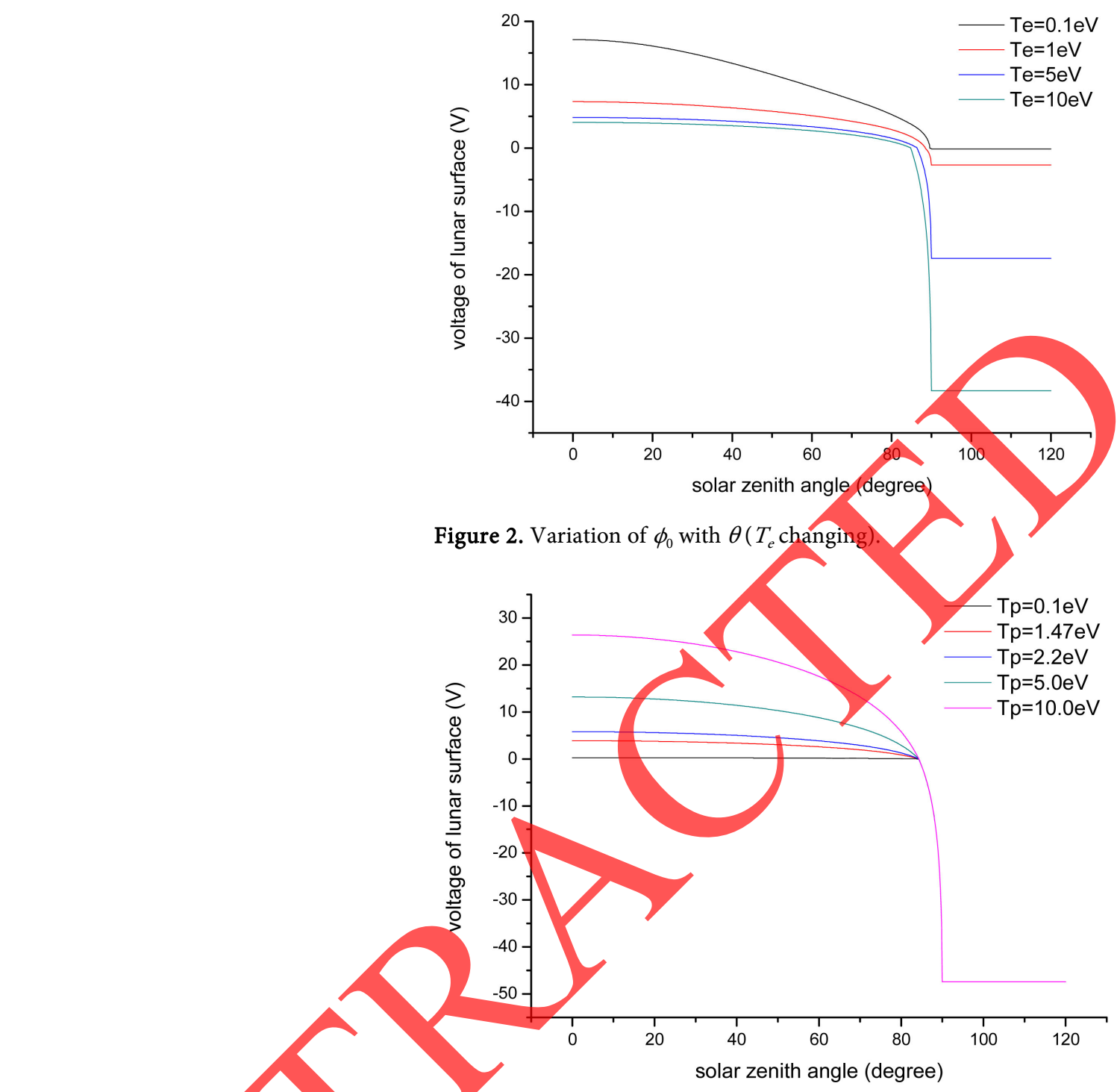

Figure 3. Variation of $\phi_{0}$ with $\theta$ ( $T_{p}$ changing).

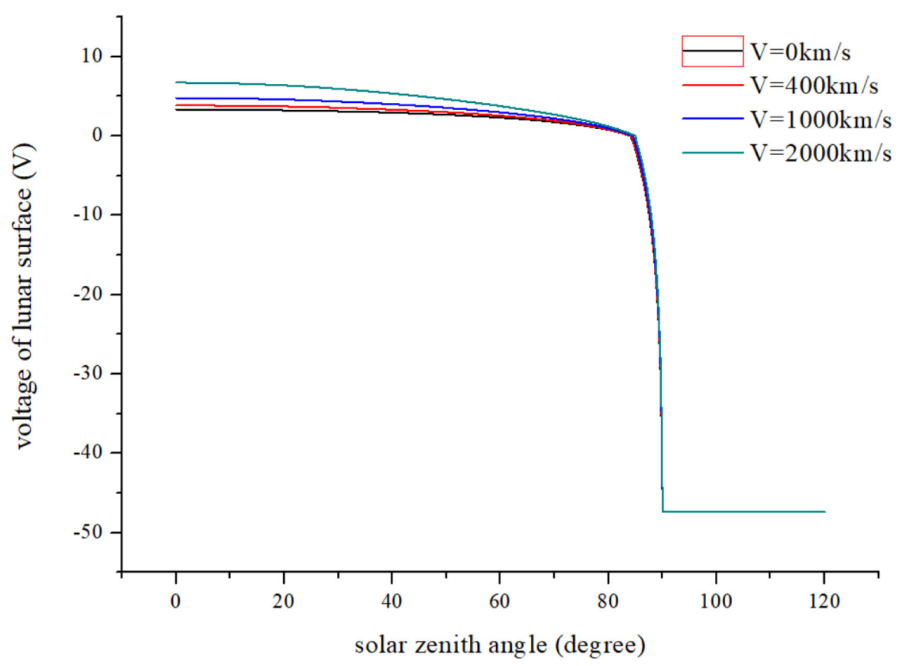

Figure 4. Variation of $\phi_{0}$ with $\theta$ ( $V$ changing). 


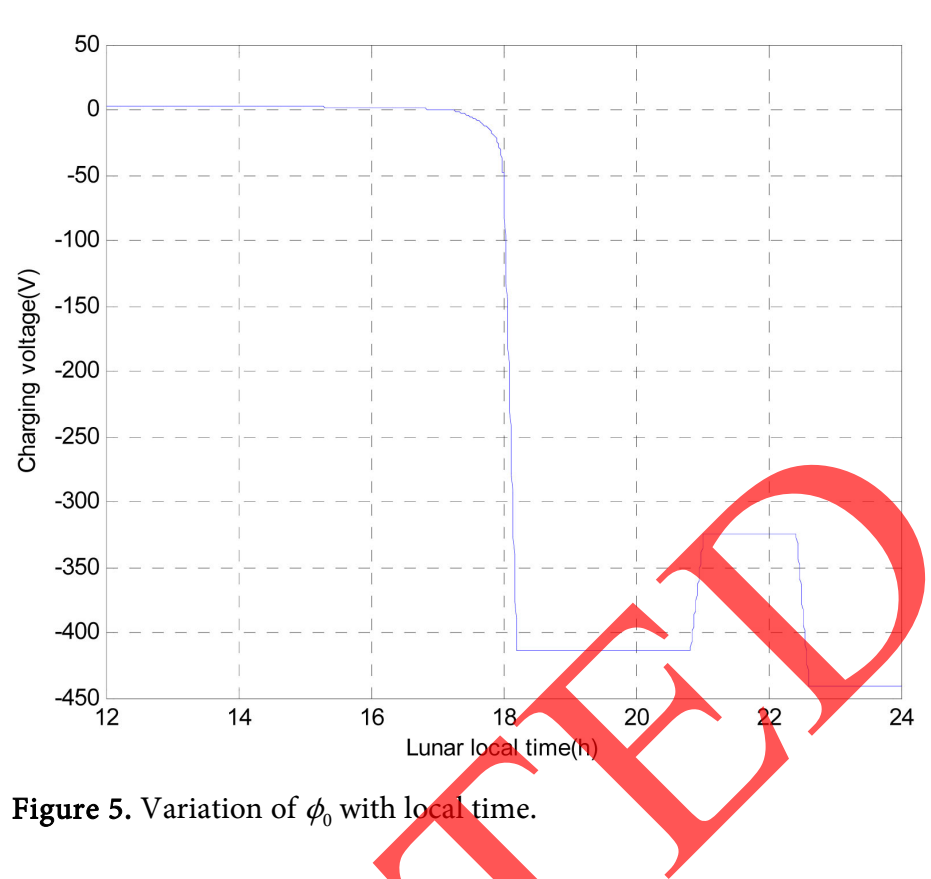

Table 1. Parameters and value used for numerical calculation.

\begin{tabular}{|c|c|c|c|c|c|}
\hline & Plasma environment & Solar wind & Lunar wake & Magneto sheath & Magneto tail \\
\hline \multirow{6}{*}{ Parameters } & $n_{0}\left(\mathrm{~cm}^{-3}\right)$ & 10 & 0.1 & 20 & 0.15 \\
\hline & (K) & & $1.276 \times 10^{6}$ & $1 \times 10^{6}$ & $1.682 \times 10^{6}$ \\
\hline & & $1.0 \times 10^{5}$ & $1.276 \times 10^{6}$ & $1 \times 10^{6}$ & $7.076 \times 10^{6}$ \\
\hline & & 400 & 20 & 350 & 72 \\
\hline & $\left.m^{2}\right)$ & 4.5 & 4.5 & 4.5 & 4.5 \\
\hline & & $1.7 \times 10^{4}$ & $1.7 \times 10^{4}$ & $1.7 \times 10^{4}$ & $1.7 \times 10^{4}$ \\
\hline
\end{tabular}

\section{Conclusions}

Based on the current balance equation, a one-dimensional charging model is established in this paper, and the variation law of the lunar surface potential under different light radiation conditons and plasma environment conditions is calculated. The calculation results show that with the increase of the solar zenith angle, the lunar surface charging potential gradually transits from positive potential to negative potential in the day and terminator area, then reaches the maximum negative potential in the moon night. With the increase of plasma density, the electron temperature, photoelectron temperature and plasma overall velocity, the charging voltage in the day side gradually decreases, and the charging voltage in the night area of the moon surface is greatly affected by the electron temperature, and the higher the electron temperature is, the more negative the charging voltage is.

According to the relative position of the moon and the earth, the plasma environment experienced by the moon includes solar wind, lunar wake, magnetosheath, magnetotail, magnetic sheath and then solar wind again. As the plasma environment is changing and transforming gradually in the lunar night side, the 
charging potential will also change all the time. When passing through different plasma environment, the charging potential may also change suddenly in a short time.

It should be noted that the charging phenomenon under the real condition of the lunar surface is much more complicated than the results of the model calculated and analyzed. The main reason is that the plasma parameters of the lunar surface are dynamically changing, which results in the charging voltage of the actual lunar surface changing all the time, and the flat curve obtained by fixed parameters in this paper will not occur in reality. In this paper, when dealing with the transition region of different plasma environment, a linear approximation is made, and the time corresponding to the transition region is not accurate. Considering the lack of lunar plasma environment model, the proportion of time for different plasma environment experienced by the lunar surface is used. In fact, in a lunar day, the time when the moon experiences magnetic sheath and magnetic tail plasma will also change. Therefore, there are some limitations in the calculation of this paper. Later, we will modify the model to consider the secondary electron emission caused by higher energy electrons. We will also try to use the dynamic plasma parameters as the calculation input to obtain more reasonable and accurate results, which will provide theoretical basis for the study of the natural phenomena of the lunar surface, and provide guidance and support for the future lunar exploration activities.

\section{Acknowledgements}

Project supported by the National Natural Science Foundation of China (Grant No.41604158).

\section{Conflicts of Interest}

The authors declare no conflicts of interest regarding the publication of this pa-

\section{References}

[1] Singer, S.F. and Walker, E.H. (1963) Photoelectric Screening of Bodies in Interplanetary Space. 1, 7-12. https://doi.org/10.1016/0019-1035(62)90004-0

[2] Grobman, W.D. and Blank, J.L. (1969) Electrostatic Potential Distribution of the Sunlit Lunar Surface. Journal of Geophysical Research, 74, 3943-3951. https://doi.org/10.1029/JA074i016p03943

[3] Freeman, J.W. and Ibrahim, M. (1975) Lunar Electric Fields, Surface Potential and Associated Plasma Sheaths. Moon, 14, 103-114. https://doi.org/10.1007/BF00562976

[4] Halekas, J.S., Mitchell, D.L., Lin, R.P., Hood, L.L., Acuña, M.H. and Binder, A.B. (2002) Evidence for Negative Charging of the Lunar Surface in Shadow. Geophysical Research Letters, 29, 1435. https://doi.org/10.1029/2001GL014428

[5] Halekas, J.S., Delory, G.T., Lin, R.P., Stubbs, T.J. and Farrell, W.M. (2008) Lunar Prospector Observations of the Electrostatic Potential of the Lunar Surface and Its Response to Incident Currents. Journal of Geophysical Research, 113, A09102. https://doi.org/10.1029/2008JA013194 
[6] Halekas, J.S., Delory, G.T., Brain, D.A., Lin, R.P., Fillingim, M.O., Lee, C.O., et al. (2007) Extreme Lunar Surface Charging during Solar Energetic Particle Events. 34, 346-358. https://doi.org/10.1029/2006GL028517

[7] Halekas, J.S., Delory, G.T., Lin, R.P., Stubbs, T.J. and Farrell, W.M. (2009) Lunar Surface Charging during Solar Energetic Particle Events: Measurement and Prediction. Journal of Geophysical Research: Space Physics. https://doi.org/10.1029/2009JA014113

[8] Manka, R.H. (1973) Plasma and Potential at the Lunar Surface. In: Grard, R.J.L., Ed., Photon and Particle Interactions with Surfaces in Space, D. Reidel Publishing Co., Dordrecht, 347-361. https://doi.org/10.1007/978-94-010-2647-5 22

[9] Stubbs, T.J., Halekas, J.S., Farrell, W.M. and Vondrak, R.R. (2007) Lunar Surface Charging: A Global Perspective Using Lunar Prospector Data. Dust in Planetary Systems, 1280, 181-184.

[10] Gold, T. (1962) Processes on the Lunar Surface. Symposium-International Astronomical Union, 14, 433-439. https://doi.org/10.1017/S0074180900178428

[11] Perkins, D. (1976) Analysis of LEAM Experiment Response to Charged Particles. Final Report Bendix Corp. ann Arbor Mi. aerospace Systems Div.

[12] Bailey, C.L. and Frantsvog, D.J. (1977) Response of the LEAM Detector to Positively Charged Microparticles. NASA Contract NAS5-23557, Concordia Coll., Moorhead, Minn.

[13] Berg, O.E., Wolf, H. and Rhee, J. (1976) Lunar Soil Movement Registered by the Apollo 17 Cosmic Dust Experiment. Lecture Notes in Physics, 48, 233-237. https://doi.org/10.1007/3-540-07615-8_486

[14] Gaier, J.R. (2005) The Effects of Lunar Dust on EVA Systems during the Apollo Missions, NASA/TM-2005-213610, NASA Glenn Research Center, Cleveland, Ohio.

[15] Stubbs, T.J., Vondrak, R.R. and Farrell, W.M. (2007) Impact of Dust on Lunar Ex-

ploration. Dust in Planetary Systems, 643, 239-243.

[16] Farrell, W.M., Stubbs, T.J., Delory, G.T., Vondrak, R.R., Collier, M.R., Halekas, J.S., et al. (2008) Concerning the Dissipation of Electrically Charged Objects in the Shadowed Lunar Polar Regions. Geophysical Research Letters, 35, L19104. https://doi.org/10.1029/2008GL034785

17] Jackson, T.L., Farrell, W.M., Killen, R.M., Delory, G.T., Halekas, J.S. and Stubbs, T.J. (2011) Discharging of Roving Objects in the Lunar Polar Regions. Journal of Spacecraft and Rockets, 48, 700-704. https://doi.org/10.2514/1.51897

[18] Horányi, M., Walch, B., Robertson, S. and Alexander, D. (1998) Electrostatic Charging Properties of Apollo 17 Lunar Dust. Journal of Geophysical Research. https://doi.org/10.1029/98JE00486

[19] Stubbs, T.J., Farrell, W.M., Halekas, J.S., Burchill, J.K., Collier, M.R., Zimmerman, M.I., et al. (2014) Dependence of Lunar Surface Charging on Solar Wind Plasma Conditions and Solar Irradiation. Planetary and Space Science, 90, 10-27. https://doi.org/10.1016/j.pss.2013.07.008 